\title{
Stability of Doubly Warped Product Spacetimes
}

\author{
Lars Andersson *
}

\begin{abstract}
Nonlinear stability for a class of doubly warped spacetimes is proved. The background spacetimes have negative Einstein factors. It shown that for dimension $D \geq 11$ there is a full parameter family of solutions to the vacuum Einstein equations which has Kasner-like singularity and Friedmann like asympotics in the future. In particular, these spacetimes have crushing singularity and are globally foliated by constant mean curvature Cauchy hypersurfaces.
\end{abstract}

\section{Introduction}

The goal of this paper is to prove the existence of a full parameter family of future complete spacetimes with quiescent singularity. In the paper [1], written jointly with Mark Heinzle, we studied a class of generalized Kasner spacetimes. These are $D=$ $m+n+1$ dimensional Lorentzian doubly warped product spacetimes of the form $\mathbb{R} \times M \times N$ with line element

$$
-d t^{2}+a^{2}(t) g+b^{2}(t) h
$$

where $(M, g)$ and $(N, h)$ are $m$ - and $n$-dimensional Einstein spaces. We shall here consider only the case where $M, N$ are negative Einstein spaces.

The paper [1] showed that the spacetimes under consideration have a Kasnerlike singularity, while the future asympotics is Friedmann-like. In that paper it was demonstrated that for dimension $D \geq 10$, a Kaluza-Klein dimensional reduction gives a spacetime with eternal acceleration. Further, and most importantly for the

Lars Andersson

Department of Mathematics, University of Miami, Coral Gables, FL 33124, USA and Albert Einstein Institute, Am Mühlenberg 1, 14476 Golm, Germany, e-mail: larsa@ math.miami.edu

* Supported in part by the NSF, with grants DMS-0407732 and DMS-0707306 to the University of Miami. 
application in the present paper, it was demonstrated in [1] that the singularity allows AVTD deformations exactly when $D \geq 11$. This is the case we consider here.

In a recent paper of the author with Vincent Moncrief [4], we proved the nonlinear stability to the future for a class of Lorentz cone spacetimes. The background spaces considered are Lorentz cones over negative Einstein spaces. The work in [4] allows general dimension and further allows the Riemannian Einstein factor to have a non-trivial Einstein moduli space. A $3+1$ dimensional case of this situation was considered in [4], there rigidity was assumed. This condition has later been removed in the $3+1$ dimensional case by Martin Reiris [10].

In the present paper, we apply the results of [3] as well as the analysis in [1], together with the Fuchsian techniques developed with Alan Rendall in [5], see also [7] for the higher dimensional case, to demonstrate for $D \geq 11$, the existence of a full parameter family of nonlinearly stable deformations of the generalized Kasner spacetimes studied in [1]. These deformations have dynamics and geometry which is controlled globally from the singularity to the infinite future. In particular, they have crushing singularity, admit a global foliation by CMC Cauchy hypersurfaces, and are future causally geodesically complete. This is the first result of its type.

\section{Warped Product Spacetimes}

Let $\left(M, \gamma^{M}\right),\left(N, \gamma^{N}\right)$ be $m$ - and $n$-dimensional compact negative Einstein spaces with

$$
\operatorname{Ric}_{\gamma^{M}}=-(m+n-1) \gamma^{M}, \quad \operatorname{Ric}_{\gamma^{N}}=-(m+n-1) \gamma^{N} .
$$

We consider line elements on a $D=m+n+1$ dimensional spacetime $\mathbb{R} \times M \times N$ of the form

$$
-d t^{2}+a^{2}(t) \gamma^{M}+b^{2}(t) \gamma^{N} .
$$

The vacuum Einstein equations for the line elements of the form (1) were analyzed in [1] in terms of scale invariant variables. Let

$$
p=-\frac{\dot{a}}{a}, \quad q=-\frac{\dot{b}}{b} .
$$

The mean curvature $\tau$ is given by

$$
\tau=m p+n q .
$$

The scale invariant variables are $^{2}$

$$
P=\frac{p}{\tau}, \quad Q=\frac{q}{\tau}, \quad A=-\frac{1}{a \tau}, \quad B=-\frac{1}{b \tau} .
$$

2 In [1], mean curvature is denoted by $H$ and the time variable $\tau$ used there is related to the proper time by $\partial_{\tau}=H^{-1} \partial_{t}$. With this definition, $T / \tau$ is a negative constant. Here, we use notation adapted to [2]. 
Here $A, B$ may be viewed as curvature variables. Define the scale invariant time coordinate $T$ by

$$
\partial_{T}=-\tau^{-1} \partial_{t}=-\tau \partial_{\tau}
$$

The Einstein equations in terms of the time $T$ for $P, Q, A, B$ forms an autonomous system, and the evolution equation for $\tau$ decouples.

The phase space of the scale invariant system resulting from the Einstein equations for the line element (1), with $M, N$ negative Einstein spaces, is a topologically a disc. There are five equilibrium points, four of which $\left(\mathrm{F}_{1}\right),\left(\mathrm{F}_{2}\right)$ and $\left(\mathrm{F}_{\mathrm{A}}\right),\left(\mathrm{F}_{\mathrm{B}}\right)$ are on the boundary, where $A B=0$, and one $\left(\mathrm{F}_{*}\right)$ is in the interior, where $A B>0$. See Fig. 1 for a schematic of the phase portrait for the case $D \geq 11$, in which case $\left(\mathrm{F}_{*}\right)$ is a stable node. In this note, we shall be interested only in this case.

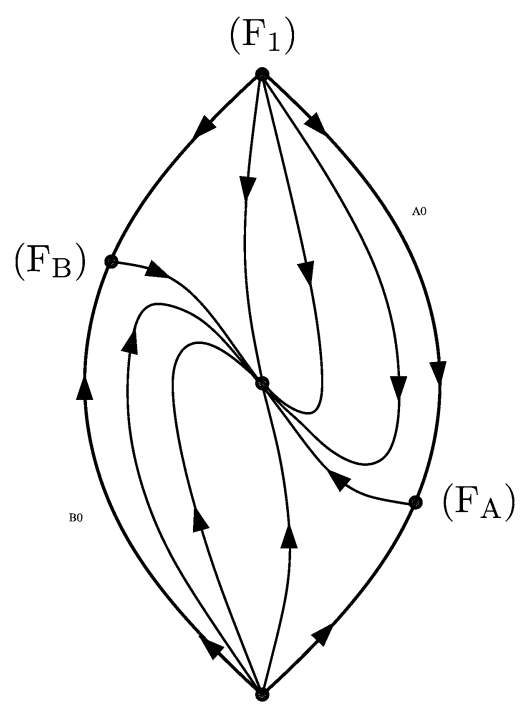

$\left(\mathrm{F}_{2}\right)$

Fig. 1 Phase portrait for $D \geq 11$, see [1]

The equilibrium points on the boundary correspond to cosmological singularities with asymptotically Kasner-like behavior. Generic past directed orbits tend to one of the two equilibrium points $\left(\mathrm{F}_{1,2}\right)$ which are characterized by the relations

$$
A=B=0, \quad m P^{2}+n Q^{2}=1, \quad m P+n Q=1,
$$

All future directed orbits tend to the equilibrium point $\left(\mathrm{F}_{*}\right)$, characterized by

$$
A=B=P=Q=\frac{1}{m+n}
$$


with asymptotically Friedmann-like behavior. In particular the spacetime geometry is asymptotic in the expanding direction to the Lorentz cone

$$
-d t^{2}+\frac{t^{2}}{(m+n)^{2}} \gamma,
$$

where

$$
\gamma=(m+n)^{2}\left(\gamma^{M}+\gamma^{N}\right)
$$

is an Einstein metric with constant $-(m+n-1) /(m+n)^{2}$ on $M \times N$. The $t$-level sets are constant mean curvature hypersurfaces, and as we shall see the warped product spacetimes under consideration are, up to a time reparametrization, in CMCSH gauge with respect to $\gamma$.

\subsection{Asymptotic Behavior}

By the results of [1, Sect. 3.4], $a, b$ have, in the case of spacetime dimension $D \geq 11$, the following asymptotic form in the expanding direction, i.e. at $\left(\mathrm{F}_{*}\right)$, as $t \nearrow \infty$,

$$
\begin{aligned}
& a=t\left[1+n c_{0} t^{-\lambda^{*}}+O\left(t^{-2 \lambda^{*}}\right)\right], \\
& b=t\left[1-m c_{0} t^{-\lambda^{*}}+O\left(t^{-2 \lambda^{*}}\right)\right]
\end{aligned}
$$

cf. [1, (3.8)], as $t \rightarrow \infty$, where $\lambda^{*}>0$ is given by

$$
\lambda^{*}=\frac{1}{2}(m+n-1-\sqrt{(m+n-1)(m+n-9)}) .
$$

The induced metric on the Cauchy surface $M \times N$ at time $t$ is given by

$$
a^{2}(t) \gamma_{M}+b^{2}(t) \gamma_{N}
$$

The Christoffel symbol of this metric contains no mixed terms, and due to the scale invariance of the Christoffel symbol it is identical to the Christoffel symbol of the metric (3). It follows that the tension field defined with respect to the metric (5) and $\gamma$ vanishes and hence (1) is automatically in CMCSH gauge with respect to the background metric $\gamma$. The second fundamental form is

$$
p a^{2} \gamma_{i j}^{M}+q b^{2} \gamma_{i j}^{N}
$$

and from (4), we have

$$
\tau=-(m+n) t^{-1}+O\left(t^{-1-2 \lambda^{*}}\right) .
$$

Recall from [4, Sect. 4] that the scale invariant Cauchy data $(g, \Sigma)$ are related to the metric and second fundamental form by 


$$
g_{i j}=\tau^{2} \tilde{g}_{i j}, \quad \Sigma_{i j}=\tau\left(\tilde{K}_{i j}-\frac{\tau}{n+m} \tilde{g}_{i j}\right) .
$$

Let $(\hat{g}, \hat{\Sigma})$ be the scale invariant data corresponding to the metric (5) and the second fundamental form (6). A calculation using (4) gives

$$
\begin{aligned}
\hat{\Sigma} & =t^{-\lambda^{*}}\left(1-\lambda^{*}\right) c_{0}\left[n \gamma^{M}-m \gamma^{N}\right]+O\left(t^{-2 \lambda^{*}}\right), \\
\hat{g}-\gamma & =t^{-\lambda^{*}}(m+n)^{2} c_{0}\left[n \gamma^{M}-m \gamma^{N}\right]+O\left(t^{-2 \lambda^{*}}\right) .
\end{aligned}
$$

The scale invariant time is $T=-\log (-\tau /(m+n))$. Due to the fact that there are no spatial degrees of freedom in $(\hat{g}, \hat{\Sigma})$, the asymptotic form the Cauchy data given in (7) immediately allows us to estimate the Sobolev distance of the scale invariant data $(\hat{g}, \hat{\Sigma})$ from the background data $(\gamma, 0)$. We have for any $s \geq 1$,

$$
\|\hat{g}-\gamma\|_{H^{s}}+\|\hat{\Sigma}\|_{H^{s-1}}=O\left(e^{-\lambda^{*} T}\right)
$$

or equivalently

$$
\|\hat{g}-\gamma\|_{H^{s}}+\|\hat{K}+\gamma\|_{H^{s-1}}=O\left(e^{-\lambda^{*} T}\right),
$$

where $\hat{K}=\tau \tilde{K}$ is the scale invariant second fundamental form. Similarly, inserting the calculated form of $\hat{\Sigma}, \hat{g}-\gamma$ into the energies $E_{S}$, we have for $s \geq 1$,

$$
E_{s}=O\left(e^{-2 \lambda^{*} T}\right)
$$

which tends to zero as $T \rightarrow \infty$.

Next we consider the asymptotic behavior of a generic orbit in the collapsing direction, asymptotic to one of the equilibrium points $\left(\mathrm{F}_{1}\right),\left(\mathrm{F}_{2}\right)$. A generic solution is of the form

$$
a=a_{0} t^{P}\left[1+O\left(t^{\delta}\right)\right], \quad b=b_{0} t^{Q}\left[1+O\left(t^{\delta}\right)\right]
$$

for constants $a_{0}, b_{0}, P, Q$, and for some $\delta>0$. Here $P, Q$ are solutions to

$$
m P+n Q=1, \quad m P^{2}+n Q^{2}=1 .
$$

It follows from these equations that $|P|<1,|Q|<1$.

\section{Fuchsian Method}

The generalized Kasner exponents can be defined as the eigenvalues of the scaleinvariant second fundamental form $K_{i}{ }^{j}$, with one index raised using the scale invariant spatial metric $g_{i j}$. By [1, Theorem 3.1] and [1, Proposition 3.2], $t=0$ corresponds to a cosmological singularity, in the sense that $a(t), b(t) \rightarrow 0$, as $t \rightarrow 0$ so that the induced metric on the $t$-level sets collapses. Further, the singularity at $t=0$ is Kasner like, i.e. the generalized Kasner exponents converge as $t \rightarrow 0$. 
The Fuchsian method allows one to construct families of solutions to the Einstein equations, with controlled behavior at a cosmological singularity, under condition that the behavior at the singularity is asymptotically velocity dominated. This means roughly that asymptotically at the singularity, spatial derivatives become insignificant and the dynamics of the Einstein equations approaches that of a family of Bianchi I spacetimes, one for each spatial point. This method was first applied to Gowdy models by Kichenassamy and Rendall [9]. The first treatment of case of the Einstein equations without symmetry was given by Andersson and Rendall [5], for the $3+1$ dimensional Einstein equations minimally coupled with a massless scalar field. The application of the Fuchsian method to the vacuum Einstein equations in $D=d+1$ spacetime dimensions was carried out by Damour, Henneaux, Rendall and Weaver [7].

The Fuchsian method applies a singular version of the Cauchy-Kowalevski theorem, and therefore requires the velocity dominated data to be real analytic in the spatial direction. Although real analytic functions are dense in $C^{\infty}$ in a compact manifold this is an important restriction, and it is desirable to generalize the results discussed here to cover smooth data or data with finite regularity. In particular it is important to prove a stability type result that shows that there is an open set of initial data, in Sobolev norm, which leads to spacetimes with asymptotically velocity dominated behavior. This was shown by Ringstrom for the Gowdy case, see [11].

The main result of the Fuchsian analysis in the case we are interested in, is that given a velocity dominated solution which satisfies certain restrictions, see below, there is a unique solution to the vacuum Einstein equations, which has the same asymptotic generalized Kasner exponents at each point as those of the velocity dominated solution. Further, the solution produced by the Fuchsian method is asymptotic to the velocity dominated solution at the singularity.

Let $p_{1} \leq p_{2} \leq \cdots \leq p_{d}$ be limiting Kasner exponents as $t \rightarrow 0$, in increasing order. By [1, Sect. 4], for $D \geq 11$, the generic spacetime of the form (1) has generalized Kasner exponents whose limit at the singularity satisfy

$$
1+p_{1}-p_{d}-p_{d-1}>0 \text {. }
$$

This is precisely those doubly warped product spacetimes which are past asymptotic to one of the equilibrium points $\left(F_{1,2}\right)$. According to the results of [7], see in particular [7, Sect. 3], this implies that one may apply the Fuchsian method to construct a full parameter family of non-homogeneous spacetimes deforming the warped product ones.

\subsection{Velocity Dominated Equations}

In order to construct the approximating spacetime, we must specify a "velocity dominated" seed solution. This is defined from initial data $\left({ }^{0} g_{i j},{ }^{0} K_{i}{ }^{j}\right)$ which satisfy the velocity dominated constraint equations [5], see [7, Sect. 2.3] for the case of space- 
times of general dimension. These are in the vacuum case,

$$
\begin{gathered}
-{ }^{0} K_{i}{ }^{j 0} K^{j}{ }_{i}+\left(\operatorname{tr}{ }^{0} K\right)^{2}=0, \\
{ }^{0} \tilde{\nabla}_{j}{ }^{0} K^{j}{ }_{i}-{ }^{0} \tilde{\nabla}_{i}\left(\operatorname{tr}{ }^{0} K\right)=0
\end{gathered}
$$

a truncated form of the Einstein vacuum constraints. By a modification of the conformal method, one may construct a full parameter family of solutions to the velocity dominated constraints.

The velocity dominated solution is constructed by solving the velocity dominated evolution equations

$$
\begin{aligned}
\partial_{t}{ }^{0} g_{i j} & =-2^{0} K_{i j}, \\
\partial_{t}{ }^{0} K^{i}{ }_{j} & =\left(\operatorname{tr}^{0} K\right)^{0} K^{i}{ }_{j} .
\end{aligned}
$$

Again, this system is a truncated form of the full Einstein evolution equations. Equations (14) has, up to time-reparametrization, solutions of the form

$$
\begin{aligned}
{ }^{0} K^{a}{ }_{b}(t) & =-t^{-1} \kappa^{a}{ }_{b}, \\
{ }^{0} g_{a b}(t) & ={ }^{0} g_{a c}\left(t_{0}\right)\left[\left(t / t_{0}\right)^{2 \kappa}\right]_{b}^{c},
\end{aligned}
$$

$\operatorname{tr} \kappa=1$. For each spatial point, the equations are just the equations modelling a Bianchi I, or Kasner spacetime.

\subsection{Velocity Dominated Solution}

The velocity dominated solution which correspond to the warped product spacetimes with the Kasner-like behavior at the singularity given by (9) has spatial metric

$$
{ }^{0} \hat{g}=a_{0}^{2} t^{2 P} \gamma^{M}+b_{0}^{2} t^{2 Q} \gamma^{N},
$$

and the covariant second fundamental form is

$$
{ }^{0} \hat{K}=-t^{-1}\left(P a_{0}^{2} t^{2 P} \gamma^{M}+Q b_{0}^{2} t^{2 P} \gamma^{N}\right)
$$

There is a unique spacetime asymptotic to the velocity dominated solution at the singularity, provided (11) holds. By [1, Sect. 4] this condition is satisfied for the warped product spacetimes of the form (1), asymptotic in the past to one of the equilibrium points $\left(\mathrm{F}_{1,2}\right)$, if $D \geq 11$. The solution to the Einstein equations corresponding to the velocity dominated data (17) is, by uniqueness, the warped product spacetime with this asymptotic behavior. This is a one-parameter family of spacetimes, up to a trivial rescaling. 
Fix $a_{0}, b_{0}$ and let $(\hat{g}, \hat{K})$ be the scale-invariant data for the warped product metric with velocity dominated data given by (17). The map from the velocity dominated data $\left({ }^{0} g\left(t_{0}\right),{ }^{0} K\left(t_{0}\right)\right)$ to the data $(\tilde{g}, \tilde{K})$ at $t_{0}$ for the vacuum spacetime constructed by the Fuchsian method with seed solution $\left({ }^{0} g,{ }^{0} K\right)$ is continuous at the background data velocity dominated data $\left({ }^{0} \hat{g},{ }^{0} \hat{K}\right)$, in terms of the natural topology on the space of real analytic tensors on $M$. See [5, 7] for details, and in particular [6] for analytical details concerning the singular form of the Cauchy-Kowalewski theorem.

The construction gives a full parameter set of real analytic spacetimes close to the background spacetime. The topology on the space of analytic functions is finer than the $H^{s}$ Sobolev topology for any $s$. Hence, the scale invariant data $(g, \Sigma)$ are $H^{s}$ close to $(\hat{g}, \hat{\Sigma})$. We summarize this discussion in the following lemma.

Lemma 1. Fix $t_{0}>0$. For each $s \geq 1, \epsilon>0$, there is a full parameter set of velocity dominated data $\left({ }^{0} g\left(t_{0}\right),{ }^{0} K\left(t_{0}\right)\right)$ close to the background velocity dominated data of the form (17), such that

$$
\left\|g\left(t_{0}\right)-\hat{g}\left(t_{0}\right)\right\|_{s}+\left\|K\left(t_{0}\right)-\hat{K}\left(t_{0}\right)\right\|_{s-1}<\epsilon,
$$

where $(g, K)$ are scale invariant data for the vacuum spacetime with velocity dominated data $\left({ }^{0} g,{ }^{0} K\right)$ and $(\hat{g}, \hat{K})$ are the scale invariant data corresponding to the warped product background metric with velocity dominated data given by (17).

\section{Stability}

The spacetime with rescaled data $(\tilde{g}, \tilde{K})$ given by Lemma 1 is not CMC foliated in the time coordinate $t$. We will now show that the spacetimes can be refoliated in CMC time, with the small data condition still valid.

Lemma 2. Assume the Einstein spaces $M, N$ are stable, with smooth deformation spaces. Fix $\tau_{0}<0$. For each $s \geq 1, \epsilon>0$, there is a $t_{0}>0$ and a full parameter set of velocity dominated data $\left({ }^{0} g\left(t_{0}\right),{ }^{0} K\left(t_{0}\right)\right)$ close to the background velocity dominated data of the form (17), such that the spacetime produced by the Fuchsian method from the velocity dominate data contains a CMC foliation with mean curvature $\tau$ taking values in an interval containing $\left(-\infty, \tau_{0}\right)$, and such that at mean curvature time $\tau_{0}$ we have

$$
\left\|g\left(\tau_{0}\right)-\hat{g}\left(\tau_{0}\right)\right\|_{s}+\left\|K\left(\tau_{0}\right)-\hat{K}\left(\tau_{0}\right)\right\|_{s-1}<\epsilon,
$$

where $(g, K)$ are scale invariant data for the vacuum spacetime with velocity dominated data $\left({ }^{0} g,{ }^{0} K\right)$ and $(\hat{g}, \hat{K})$ are the scale invariant data corresponding to the warped product background metric with velocity dominated data given by (17). Further, $(g, K)$ are satisfy the CMCSH gauge condition and the shadow metric condition with respect to the background metric $\gamma$ on $M \times N$.

Proof. The spacetime produced by the Fuchsian method is asymptotically Kasnerlike at the singularity, and in particular the singularity is crushing. Recall that the 
time $t_{0}$ in Lemma 1 is arbitrary. The mean curvature for the warped product background metric takes all values in $(-\infty, 0)$ and for velocity dominated data sufficiently close to the background data, we have by a suitable choice of $t_{0}$ in Lemma 1 , $\operatorname{tr}_{\tilde{g}} \tilde{K}\left(t_{0}\right)>\tau_{0}$. This means that the Cauchy surface at time $t_{0}$ is a barrier for the mean curvature equation, and hence by [8], there is a CMC foliation with mean curvature taking values in an interval containing $\left(-\infty, \tau_{0}\right)$. Due to the elliptic nature of the CMC equation, the smallness condition (18) is satisfied for velocity dominated data sufficiently close to the background.

By assumption the Einstein spaces $M, N$ are stable, with smooth deformation space. By [4, Sect. 3] that this is true also for $(M \times N, \gamma)$. By the work in [4, Sect. 4.2], we may after possibly decreasing $\epsilon$ and acting on the background warped product metric with a time-independent diffeomorphism, assume that the data produced above satisfies the CMCSH gauge and the shadow-metric condition with respect to an Einstein metric $\bar{\gamma}$ close to $\gamma$ and in the deformation space $\mathcal{V}$ of $\gamma$. Due to the local uniformization result [4, Proposition 2.4] $\bar{\gamma}$ is of the form (3) with respect to two Einstein metrics $\bar{\gamma}^{M}, \bar{\gamma}^{N}$ close to $\gamma^{M}, \gamma^{N}$. The Einstein equations for the warped product spacetimes are independent of the choice of Einstein metric. Hence this modified background has the same dynamics as the original one and we may without loss of generality assume the CMCSH and shadow metric condition is satisfied with respect to the original background data. This completes the proof of the lemma.

We are now ready to apply the above construction of a CMC foliated, quiescent spacetime, together with the main result of [4], to prove the existence of a full parameter family of quiescent, future complete spacetimes. In Sect. 2.1 we proved that the data $(\hat{g}, \hat{\Sigma})$ corresponding to the warped product background spacetime tends, in Sobolev norm, to the data corresponding to the Lorentz cone metric (2). Together with the smallness estimate in Lemma 2 this means that by suitable choosing $\tau_{0}, \epsilon$, the data for the quiescent spacetime constructed by the Fuchsian method satisfies, at CMC time $\tau_{0}$, the conditions of the stability theorem [4, Theorem 7.1]. This gives

Theorem 3. Assume $M, N$ are stable with smooth deformation space. Further, assume $D \geq 11$. Then, there is a full parameter set of velocity dominated data $\left({ }^{0} g,{ }^{0} K\right)$ close to the velocity dominated data $\left({ }^{0} \hat{g},{ }^{0} \hat{K}\right)$, corresponding to a warped product spacetime of the form (1), with a Kasner-like initial singularity with generalized Kasner exponents close to $P, Q$ satisfying equation (10), such that the quiescent spacetime constructed using the Fuchsian method admits a global CMC foliation, and is asymptotic to the Lorentz cone spacetime with line element (2).

\section{References}

1. L. Andersson and J.M. Heinzle, Eternal acceleration from M-theory. Adv. Theor. Math. Phys. 11(3), 371-398 (2007)

2. L. Andersson and V. Moncrief, Elliptic-hyperbolic systems and the Einstein equations. Ann. Henri Poincaré 4(1), 1-34 (2003) 
3. L. Andersson and V. Moncrief, Future Complete Vacuum Spacetimes, The Einstein Equations and the Large Scale Behavior of Gravitational Fields, pp. 299-330. Birkhäuser, Basel (2004)

4. L. Andersson and V. Moncrief, Einstein spaces as attractors for the Einstein flow (2009, under preparation)

5. L. Andersson and A.D. Rendall, Quiescent cosmological singularities. Commun. Math. Phys. 218(3), 479-511 (2001)

6. M.S. Baouendi and C. Goulaouic, Remarks on the abstract form of nonlinear CauchyKovalevsky theorems. Commun. Partial Differ. Equ. 2(11), 1151-1162 (1977)

7. T. Damour, M. Henneaux, A.D. Rendall, and M. Weaver, Kasner-like behaviour for subcritical Einstein-matter systems. Ann. Henri Poincaré 3(6), 1049-1111 (2002)

8. C. Gerhardt, $H$-surfaces in Lorentzian manifolds. Commun. Math. Phys. 89(4), 523-553 (1983)

9. S. Kichenassamy and A.D. Rendall, Analytic description of singularities in Gowdy spacetimes. Class. Quantum Gravity 15(5), 1339-1355 (1998). Preprint at http://www.aei-potsdam. mpg.de

10. M. Reiris, Aspects of the long time evolution in general relativity and geometrizations of three-manifolds (2005). gr-qc/0510102

11. H. Ringström, On Gowdy vacuum spacetimes. Math. Proc. Camb. Philos. Soc. 136(2), 485$512(2004)$ 